\title{
A Novel Method for Dynamic Vehicle Routing Problem
}

\author{
Tao Ning ${ }^{1,2, *}$, Chen Guo ${ }^{1}$ and Rong Chen ${ }^{1}$ \\ ${ }^{I}$ College of Information Science and Technology, Dalian Maritime University, China; ${ }^{2}$ College of Software, Dalian \\ Jiaotong University, China
}

\begin{abstract}
For the purpose of solving the dynamic demand of the customers in the distribution process, and reducing the distribution costs, a hybrid quantum optimization based on ant colony algorithm is proposed. A mathematical model of the dynamic scheduling problems based on the virtual multi-distribution yard is established. The quantum chromosome is constructed, and the demand of customer is updated through the quantum rotation gate by ACA. The experimental examples and the contradistinction with the existing intelligent algorithms show that the proposed method can not only improve the convergence speed of the global optimal solution but enhances the fitness for the system to solve the dynamic problem and provide valid method for DVRP.
\end{abstract}

Keywords: Convergence speed, dynamic vehicle routing problem, hybrid quantum algorithm.

\section{INTRODUCTION}

The vehicle routing problem (VRP) proposed by Danzig and Ramser in 1959 has become a kind of classic combination and optimization problems in operations research [1]. The purpose of the problem is to achieve the optimization of transport costs through constructing a suitable vehicle schedule. There are a lot of uncertainty existing in VRP, such as the customer needs, the transportation needs, subjective awareness of the path-makers, traffic condition and vehicle condition, all of the above need the schedule administrator to make a correct response on the updated information in a short time with the scheduling system, and need the schedule administrator to make a correct response on the updated information in a short time with the scheduling system, and modify to generate a new schedule planning. This kind of VRP is called Dynamic Vehicle Routing Problem (DVRP) [2]. Psaraftis [3] defined the DVRP as "Arrangements for the vehicle scheduling to meet the need of customers real-time travel" in 1988. Literature [4] defined the DVRP as "In the case of system information is updated in real time, arrangements for the vehicle scheduling to achieve the system goal". The domestic and foreign researchers used a variety of methods to solve the dynamic vehicle scheduling problems, and achieved certain results in the aspects of model and algorithm.

Literature [5] studied the random demands VRP with nerve the dynamic programming method to solve the single DVRP, the studied model has been applied into the field of postal and express delivery, distribution and production, etc. Literature [6] studied VRP considering the traffic information, real time information of new customers using branch and bound method and established the constraint model.

*Address correspondence to this author at the College of Information Science and Technology, Dalian Maritime University, and College of Software, Dalian Jiaotong University, China; Tel/Fax: 8613940901029;

E-mail: daliannt1@126.com
Literature [7] studied the DVRP based on dynamic demands and travel time, and discussed the processing of dynamic demands. Literature [8] changed the DVRP into SVRP through introducing the virtual task point.

The objective function of DVRP is more complicated than that of SVRP (Static vehicle routing problem). The model of DVRP can be described as follows: There are one yard and m nodes (customer node) to be served in some transport net; the vehicles start from the yard and will return to the yard after having served some customer nodes. The customers can be divided into deterministic demand customers and the uncertainty of customer according to the different demand. The transport net is SVRP when the demand of customer has not changed, otherwise, the scheduling system will modify the formulated schedule plan, and such is called DVRP, as shown in Fig. (1).

The transport capacity of each vehicle is expressed as C, the demand of each node is expressed as fuzzy number D, the distance between node $\mathrm{i}$ and node $\mathrm{j}$ as $c_{i j}$, and to solve the vehicle schedule problem with the least transport cost. The objective is to minimize the number of vehicles.

\section{MATHEMATICS MODELS OF DVRP}

The DVRP studied here includes one distribution center with a team of vehicles whose type is all the same, customers sets, each customer can be served by only one vehicle. The symbol of model is as follows: the number of distribution center is 0 , the number of customer is $1,2, \ldots, N$, the number of vehicles in distribution center are $1,2, \ldots, K$, the maximum load of the vehicle is $Q$, the cumulative weight of vehicle $\mathrm{k}$ started for node $i$ is $q_{i k}(t)(i=1,2, \ldots, \mathrm{N})$ and $q_{i k}(t)<Q, q_{i}$ is the demand of customer $I$, the fixed cost of vehicles is $F_{k}$ $(k=1,2, \ldots, \mathrm{K})$, the travel time of the vehicle from node $i$ to node $j$ is $t_{i j}(i, j \in\{1,2, \ldots, \mathrm{N}\})$, the distance between node $i$ and node $j$ is $d_{i j}(i, j \in\{1,2, \ldots, \mathrm{N}\})$, the cost from node $i$ to node $j$ is $c_{i j}(i, j \in\{1,2, \ldots, \mathrm{N}\})$, the transport weight for vehicle $k$ 

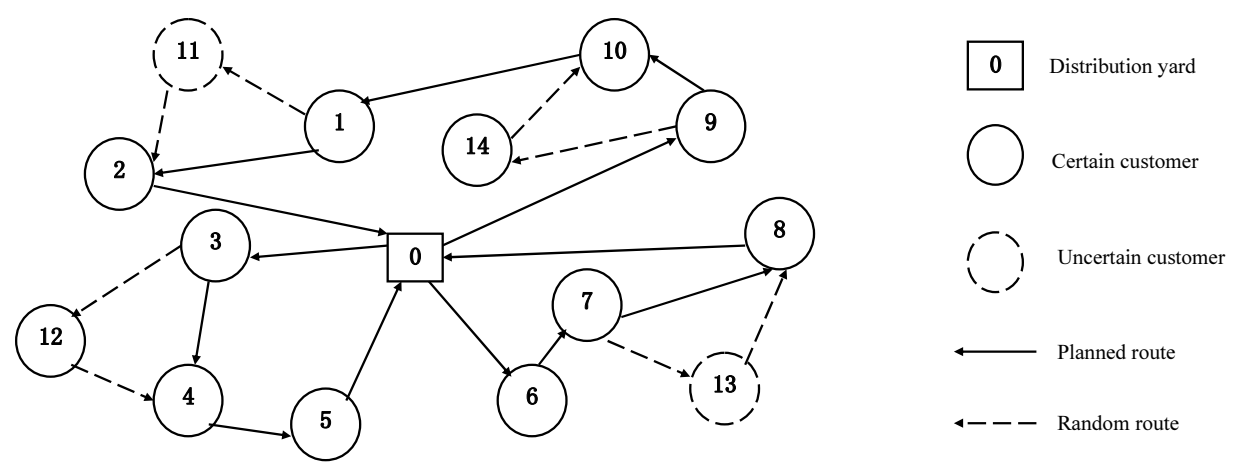

Fig. (1). Dynamic transport net.

from node $i$ to node $j$ is $w_{i j k}(k=1,2, \ldots, \mathrm{K}, i, j \in\{1,2, \ldots, \mathrm{N}\})$, the service time for vehicle $\mathrm{k}$ at node $i$ is $s_{k i}$ $(k=1,2, \ldots, \mathrm{K} ; i=1,2, \ldots, \mathrm{N})$.

In the case of vehicle load allowed, add the new customer demands into the existing schedule, if the schedule is within the formulated plan, another new vehicle should be arranged to serve. If the demands of original customers increases and exceeds the maximum vehicle load, then select the customers node of the last service in this sub-path as a new customer needs treatment, until the car limit is met. If the customer node is looked as a distribution yard, the original single distribution path is equivalent to the multi-distribution path problem. Assume that $\mathrm{W}$ is the total amount of demand including the not served customers in static phase and the new customer demands in dynamic phase, with the number of $1,2, \ldots, \mathrm{W}$; $\mathrm{M}$ indicates the number of customer nodes as a new distribution yard, with the number of $\mathrm{N}+1, \mathrm{~N}+2, \ldots$, $\mathrm{N}+\mathrm{M}$, then the number of original yard will change into $\mathrm{N}+\mathrm{M}+1$, the increased number of car is $\mathrm{L}$. The vehicle started from the distribution yard in the static phase locates at node $i$, with the number of $\mathrm{W}+i$; the remainder of the vehicles in the static phase is $Q-q_{i k}(t)$.

Definition of decision variables:

$$
\begin{aligned}
x_{i j k} & =\left\{\begin{array}{l}
1, \text { vehicle } \mathrm{k} \text { from } \mathrm{i} \text { to } \mathrm{j} \text { has dynamic demand } \\
\text { 0otherwise }
\end{array}\right. \\
y_{i k} & =\left\{\begin{array}{l}
1, \text { the demand of node } \mathrm{i} \text { is met by vehicle } \mathrm{k} \\
\text { 0otherwise }
\end{array}\right.
\end{aligned}
$$

The mathematics model of DVRP at moment $\mathrm{t}$ is as follows:

$$
\begin{aligned}
& \min Z=\sum_{k=1}^{K} \sum_{i=1}^{N+M+1} \sum_{j=1}^{N+M+1} c_{i j} x_{i j k}+\sum_{k=K+1}^{K+L} \sum_{i=1}^{N+M+1} \sum_{j=1}^{N+M+1} c_{i j} x_{i j k}+ \\
& \sum_{k=K+1}^{K+L} F_{k} \sum_{j=1}^{N} x_{(N+M+1) j k} \\
& \text { s.t. } \sum_{k=1}^{K+L} y_{i k}=1, \forall i \\
& \sum_{j=1}^{N+M+1} x_{i j k}=y_{i k}, \forall i, k
\end{aligned}
$$

$$
\begin{aligned}
& \sum_{i=1}^{N+M+1} x_{i j k}=y_{j k}, \forall j, k ; \\
& \omega_{(N+M+1) j k}=x_{(N+M+1) j k}, \forall j, k ; \\
& \sum_{i=1}^{N+M+1} x_{i j k}\left(\omega_{i j k}-q_{j}\right) \geq 0, \forall j, k ; \\
& \sum x_{i j k} \leq|T|-1, T \subset\{1,2, \quad \mathrm{~N} \quad\}, T \neq \varnothing, \forall k ;
\end{aligned}
$$

Formula (1) is objective function, including unfinished transport cost in the static phase and the new customer, the added vehicles ones in the dynamic phase, formula (2)-(7) are the constraints, formula (2) ensures that each customer can be served; formula (3) and formula (4) ensure that each customer can only be served one time; formula (5) indicated that the amount of transport of the vehicles from the yard is equal to the amount of full load; formula (6) indicates that the vehicle has enough space for the customer before serves him, which means the vehicle is not empty; formula (7) is to eliminate the sub-loop.

\section{DESIGN OF HYBRID QUANTUM ANT COLONY ALGORITHM}

\subsection{Ant Colony Algorithm (ACA)}

Ant colony algorithm is to seek the shortest path to the food through transmitting the message with the pheromone between ant individuals, which are the optimal solution, which include three mechanisms: selection, updating, and coordination [9]. The algorithm has been applied to solve the shortest path problem, traveling salesman problems, which can reflect the superiority for solving combinatorial optimization problems: (1) strong robustness; (2) self-organization; (3) positive feedback; (4) distributed computing. However, ant colony algorithm also has its inherent defects, such as: (1) ant colony algorithm has faster calculation of the convergence rate at the beginning, but running late because of all the individual search solution is exactly the same and may cause the algorithm to a standstill, unable to obtain the global optimal solution; (2) when the ant colony is larger in scale, the randomness of ant individual search will reduce the efficiency of the algorithm. The domestic and foreign scholars have proposed the improvement strategy for the ant 
Table 1. Table of rotation gate.

\begin{tabular}{|c|c|c|c|c|c|c|c|}
\hline \multirow{2}{*}{$x(m, n)$} & \multirow{2}{*}{$b(m, n)$} & \multirow{2}{*}{$\mathrm{f}(\mathrm{x})<\mathrm{f}(\mathrm{b})$} & \multirow{2}{*}{$\theta$} & \multicolumn{4}{|c|}{$s(\alpha, \beta)$} \\
\hline & & & & $\alpha \beta>0$ & $\alpha \beta<0$ & $\alpha=0$ & $\beta=0$ \\
\hline 0 & 0 & false & 0 & 0 & 0 & 0 & 0 \\
\hline 0 & 0 & true & 0 & 0 & 0 & 0 & 0 \\
\hline 0 & 1 & false & 0 & 0 & 0 & 0 & 0 \\
\hline 0 & 1 & true & $0.05 \pi$ & -1 & +1 & \pm 1 & 0 \\
\hline 1 & 0 & false & $0.01 \pi$ & -1 & +1 & \pm 1 & 0 \\
\hline 1 & 0 & true & $0.025 \pi$ & +1 & -1 & 0 & \pm 1 \\
\hline 1 & 1 & false & $0.005 \pi$ & +1 & -1 & 0 & \pm 1 \\
\hline 1 & 1 & true & $0.025 \pi$ & +1 & -1 & 0 & \pm 1 \\
\hline
\end{tabular}

colony algorithm, such as the combination of ant colony algorithm and genetic algorithm [10], the combination of ant colony algorithm and particle swarm optimization [11], etc.

\subsection{Quantum Algorithm (QA)}

Quantum evolutionary algorithm is to indicate the chromosome coding with the probability amplitude of qubit based on the basis of the quantum state vector representation, which make a chromosome express more than one state superposition and quantum rotation gate and the quantum NOT gate to realize the updating of chromosomes [12].

\subsubsection{Quit}

The basis storage unit is a quit in a quantum computer, which is qubit, it can be in the superposition state in addition to the state " 0 " or " 1 ". The superposition state is arbitrary linear superposition of state " 0 " and " 1 ", these two states is of certain probability. The basis state of a qubit can be expressed as $\mid 0$ land $|1\rangle$ superposition state can be indicated with the unit vector of two-dimensional Hibert space: $|\Phi\rangle=\alpha|0\rangle+\beta|1\rangle, \alpha$ and $\beta$ are plural, indicating the probability amplitude of state $\mid 0$ land $\mid 1$, what's more, $|\alpha|^{2}+|\beta|^{2}=1$.

\subsubsection{Quantum Chromosome}

When DVRP with $\mathrm{n}$ nodes will be solved, the 3dimensional quantum-bit matrix of $n \times n \times 2$ indicates the quantum chromosome, and composes the initial populations of $q(t)$ [13]. Decoding with the method of "first line, after grouping", step 1 generates the sequence of the customer service randomly and the random number for constructing 0 1 observation matrix of $n \times n$, the random number range is located in $[0,1]$ and to ensure that each row each column has only one 1 , vertical axis represents the customer number, and horizontal axis represents the service sequence.

For example, the $0-1$ matrix containing 4 customer scheduling problems is as follows:

$$
\left[\begin{array}{llll}
0 & 1 & 0 & 0 \\
0 & 0 & 1 & 0 \\
1 & 0 & 0 & 0 \\
0 & 0 & 0 & 1
\end{array}\right]
$$

Formula (8) indicates the sequence of customers is 2->3$>1->4$. The vehicle transport schedule is formed at step 2, serving in accordance with customer order each time; if the current vehicle can not meet the new customer demands, then it is needed to add a new vehicle, bit encoding can be converted to integer encoding through this way: $(0,2,3,0,1$, $4,0)$, which indicates 4 customer demands must be served by 2 vehicles, 0 is distribution yard. If the number of vehicles required yard more than the total number of vehicles, the path is infeasible solutions must generate a new qubit.

\subsubsection{Quantum Rotation Gate}

Guide individuals through the quantum gates of evolution, according to the Schrodinger equation [14] requires the design of quantum gates by the unitary transformation to control the quantum state of the evolution of the qubit implementation include: non-doors, revolving doors, Hadamard transform, doors, etc [15], the most common quantum rotation gate is as follows:

$\left[\begin{array}{c}\alpha_{(m, n)}^{t+1} \\ \beta_{(m, n)}^{t+1}\end{array}\right]=U(\delta \theta) \cdot\left[\begin{array}{l}\alpha_{(m, n)} \\ \beta_{(m, n)}\end{array}\right]=\left[\begin{array}{cc}\cos (\delta \theta) & -\sin (\delta \theta) \\ \sin (\delta \theta) & \cos (\delta \theta)\end{array}\right]\left[\begin{array}{l}\alpha_{(m, n)} \\ \beta_{(m, n)}\end{array}\right]$

$\delta$ indicates the direction of rotation, to ensure that the algorithm converges, its value in Table $1 ; \theta$ is the angle of rotation which to control the speed of convergence.

$x(m, n)$ indicates a certain element of current 0 -1 chromosome matrix, $b(m, n)$ indicates the accordance element at the current optimal chromosome, $f(x)$ is objective function. For example: when $x(m, n)=0, b(m, n)=1, f(x)<f(b)$, in order to obtain the converge the better target value, the 0 probability should be increased, when increases the value of $|\alpha|^{2}$, if $(\alpha$, $\beta$ ) is in the direction of the $1^{\text {st }}$ and $3^{\text {rd }}$ quadrants, the rotation is clockwise, otherwise, if in the direction of the $2^{\text {nd }}$ and $4^{\text {th }}$ quadrants, the rotation is counterclockwise.

\subsection{Hybrid Quantum Ant Colony Algorithm (HQACA)}

This article proposed a method of combining quantum computation and ant colony algorithm based on the analysis of advantages and disadvantages of them to solve DVRP: pheromone to represent the state vector in quantum algorithms, quantum rotation gate to update the pheromone, the qubits to store information.

The specific steps of HQACA are as follows:

(1) Initialize the quantum population $Q(p)$ : the size of population is $\mathrm{N}$, the number of quantum is, and $Q(p)=\left\{q_{1}^{p}, q_{2}^{p}, \quad q_{N}^{p}\right\}$

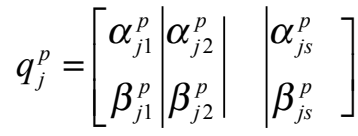


Table 2. Statistics of the distances units $(\mathbf{k m})$.

\begin{tabular}{|c|c|c|c|c|c|c|c|c|}
\hline No. & 1 & 2 & 3 & 4 & 5 & 6 & 7 & 8 \\
\hline 1 & 0 & 28.4 & 68.1 & 96.6 & 155 & 185.6 & 199.2 & 210.3 \\
\hline 2 & 28.4 & 0 & 19.5 & 15.3 & 38.2 & 60.9 & 155.9 & 200 \\
\hline 3 & 68.1 & 19.5 & 0 & 20.5 & 18.6 & 19.2 & 100 & 60.5 \\
\hline 4 & 96.6 & 15.3 & 20.5 & 0 & 44.1 & 60 & 110.5 & 80.2 \\
\hline 5 & 155 & 38.2 & 18.6 & 44.1 & 0 & 15.9 & 35.4 & 68.1 \\
\hline 6 & 185.6 & 60.9 & 19. 2 & 60 & 15.9 & 0 & 40.2 & 130.8 \\
\hline 7 & 199.2 & 155.9 & 100 & 110.5 & 35.4 & 40.2 & 0 & 40. 8 \\
\hline 8 & 210.3 & 200 & 60.5 & 80.2 & 68.1 & 130.8 & 40.8 & 0 \\
\hline
\end{tabular}

Table 3. Characteristic of mission.

\begin{tabular}{ccccccccc}
\hline Mission & 1 & 2 & 3 & 4 & 5 & 6 & 7 & 8 \\
\hline $\mathrm{Q}$ & 2 & 1.5 & 4.5 & 3 & 1.5 & 4.0 & 2.5 & 3.0 \\
$\mathrm{~T}$ & 1 & 2.0 & 1.0 & 3 & 2.0 & 2.5 & 3.0 & 0.8 \\
{$\left[\mathrm{Et}_{\mathbf{2}} \mathrm{LT}_{\mathbf{1}}\right]$} & {$[1,4]$} & {$[4,6]$} & {$[1,2]$} & {$[4,7]$} & {$[3,5.7]$} & {$[2,3]$} & {$[5,8]$} & {$[1.5,4]$} \\
\hline
\end{tabular}

$\mathrm{j}=1,2, \ldots, \mathrm{N}$. At beginning, all elements $\alpha$ and $\beta$ of $q_{j}^{1}=\left.q_{j}^{p}\right|_{p=1}$ are initialized as $1 / \sqrt{2}$;

(2) Generate the population of binary solution $P(p)$ $(\mathrm{p}=1)$ : construct $P(p)=\left\{x_{1}^{p}, x_{2}^{p}, \quad x_{N}^{p}\right\}$ according to the qubit probability $\left(\left|\alpha_{j}^{p}\right|^{2}\right.$ or $\left.\left|\beta_{j}^{p}\right|^{2}, j=1,2, \ldots, \mathrm{N}\right) \mid$ of $\mathrm{Q}(\mathrm{p})$, the binary solution $x_{j}^{p}(j=1,2, \quad, \mathrm{~N})$ is a binary string with the length of s;

(3) Initialize the parameter: initialize the number of iteration $\mathrm{p}=0$, and set the max number of loop $p_{\max }$, parameter $\alpha, \beta$ and $\gamma$, arrange the sequence of $\mathrm{s}$ ants (distribution vehicles) randomly;

(4) Ant individuals select the elements (customer nodes) according to the state transition probability, and release the pheromone;

(5) Record the optimal solution of search iteration;

(6) Updates the information of each edge with quantum rotation gate;

(7) If meet the termination condition, $p \geq p_{\max }$, then terminate the iteration, otherwise, $p=p+1$, turn to (3).

\section{NUMERICAL EXAMPLE}

\section{Example 1}

Applied the HQACA based on ACA to DVRP. The distribution system studied included seven static customer demands, one dynamic customer demand and one distribution yard. The demand of each customer indicates as $\mathrm{d}=\left[\begin{array}{lllll}1 & 2 & 1 & 2 & 1\end{array}\right.$ $\left.\begin{array}{lll}4 & 2 & 1\end{array}\right]$, there are 2 vehicles with the load capacity of 8 tons. The distance between distribution and each customer node is shown in Table 2:
Literature [16] used HGA (population size of $P_{N}=60$, crossover probability of $P c=0.8$, mutation probability of $P e=0.05)$ and $\operatorname{DPG}\left(P_{N}=30 ; P c=0.7,0.8 ; P e=0.06,0.1\right)$; literature [17] used QPSO $\left(P_{N}=60\right.$, inertia factor of $\omega=0.4$, acceleration factor of $a 1=a 2=1.49)$, calculated 20 times respectively. The average value of $\mathrm{HGA}$ was 71.15 , the known optimal solution was obtained 2 times; the average value of DPGA was 68.275 , the known optimal solution was obtained 4 times; the average value of QPSO was 62.275, the known optimal solution was obtained 10 times.

Using the above 3 methods and the novel method of HQACA $\left(P_{N}=60, \omega_{0}=1.2, \omega_{\mathrm{e}}=0.8, a 1=a 2=2, T_{0}=3, B=0.99\right.$ $T e=0.01$ ), the average value of HQACA was 65.975 , the optimal solution was obtained 19 times, the success probability was $96 \%$. It can be known that the method of HQACA is better than HGA, DPGA and QPSO in the case of the same parameters.

\section{Example 2}

The example in literature [18] with six static customer demands and two dynamic customer demands was used. The load capacity of each vehicle is $Q$, the service time is $T_{i}$, the demand time interval is $\left[E T_{i}, L T_{i}\right]$, as is shown in Table 3 .

Literature [17] used HGA $\left(P_{N}=40, P c=0.6, P e=0.2\right.$, $n_{\max }=200$, roulette wheel method to select a sub-population); literature [18] used QPSO $\left(P_{N}=40\right.$, with 2 sub-population, the size is $\left.22, \omega=0.729, a 1=a 2=1.49445, \mathrm{n}_{\max }=200\right)$. Calculated 50 times with HGA, QPSO and HQACA, the result was shown in Table 4

As was shown in Table 4 that the approximate optimal solution with the proposed algorithm can be obtained in a short period and less number of iterations in DVRP than the HGA and QPSO algorithm. It can be avoided by the local convergence of the traditional algorithm using HQACA combined ACA. Further experimental data showed that: 
Table 4. Algorithm comparison.

\begin{tabular}{|c|c|c|c|}
\hline Algorithm & Search Success Rate (\%) & Average Cost & Number of Iteration \\
\hline \hline HGA & 46 & 642.5 & 398 \\
\hline QPSO & 76 & 612.0 & 162 \\
\hline HQSAA & 91.2 & 607.2 & 158 \\
\hline
\end{tabular}

HQACA can not only converge quickly but is not easy to fall into local optimal solution.

\section{CONCLUSION}

The mathematics model of dynamic vehicle problem was established on the basis of the static vehicle scheduling problem in this article, and a novel hybrid quantum ant colony algorithm was proposed to solve this problem. The objective function and constraints of DVRP model was compared with the SVRP model, and the steps of solving problems was designed. It can be concluded from the compared results that: the proposed HQACA need less parameters and the convergence rate is faster to the DVRP of different size in the same population size and conditions, what's more, in a relatively short period of time, the global optimal solution can be obtained and then avoid the existing intelligent algorithm converges to a local optimal solution and avoided the shortcomings of converging to a local optimal solution and premature with the classic intelligent algorithm.

\section{CONFLICT OF INTEREST}

The authors confirm that this article content has no conflict of interest.

\section{ACKNOWLEDGEMENTS}

This work was partly supported by the National Natural Science Foundation, China (No.61374114), the Fundamental Research Funds for the Central Universities (DMU No. 3132014321), the Scientific Research Project of Liaoning Province Education Department, China (No. L2014183) and the Scientific Project of Dalian City (2014A11GX006).

\section{REFERENCES}

[1] G. Dantzig, and J. Ramser, "The truck dispatching problem", Management Science, vol. 6, no. 1, pp. 80-91, 1959.

[2] L. Schrage, "Formulation and structure of more complex realistic routing and scheduling problem", Networks, vol. 11, no. 2, pp. 229232,1981

[3] W. L. Wang, H. P. Huang, Y. W. Zhao, and J. 1. Zhang, "Dynamic customer demand VRP with soft time windows based on vehicle sharing", Computer Integrated Manufacturing Systems, vol. 17, no. 5, pp. 162-169, 2011.
[4] J. L. Zhang, Y. W. Zhao, and H. Y. Wang, "Modeling and algorithms for a dynamic multi-vehicle routing problem with Customers dynamic requests", Computer Integrated Manufacturing Systems, vol. 16, no. 3, pp. 543-551, 2010.

[5] B. L. Xie, Y. H. Guo, and Q. Guo, "Dynamic vehicle routing problems: status and prospect", Systems Engineer-theory Methodology Application, vol. 11, no. 2, pp. 30-34, 2002.

[6] A. L. Kok, E. W. Hans, J. M. J. Schutten, and W. H. M. Zijm,“A dynamic programming heuristic for vehicle routing with timedependent travel times and required breaks", Flexible Services and Manufacturing Journal, vol. 22, no. 1, pp. 83-108, 2010.

[7] N. Secomandi, "Comparing neuro-dynamic programming algorithms for the vehicle routing problem with stochastic demands", Computers and Operations Research, vol. 27, no. 11, pp. 1201$1225,2000$.

[8] T. Ning, Study of Application of Hybrid Quantum Algorithm in Vehicle Routing Problem, Dalian: Dalian Maritime University, 2013.

[9] K. C. Tan, Y. H. Chew and L. H. Lee, "A hybrid multi-objective evolutionary algorithm for solving vehicle routing problem with time windows", Computational Optimization and Applications, vol. 34, no. 1, pp. 115-151, 2006.

[10] K. Sundareswaran, "Line current harmonic elimination and voltage control of PWM AC/DC converter using a hybrid genetic algorithm", Electric Power Components and Systems, vol. 35, no. 2, pp. 119-133, 2007.

[11] Y. H. Hou, L. J. Lu, and X. Y. Xiong, "Enhanced particle swarm optimization algorithm and its application on economic dispatch of power systems", Proceedings of the Chinese Society of Electrical Engineering, vol. 24, no. 7, pp. 99-104, 2004.

[12] J. S. Rao, and R. Tiwari, "Optimum design and analysis of thrust magnetic bearings using multi objective genetic algorithms", International Journal for Computational Methods in Engineering Science and Mechanics, vol. 9, no. 4, pp. 223-245, 2008.

[13] Y. W. Zhao, D. J. Peng, J. L. Zang, W. L. Wang, "Quantum evolutionary algorithm for capacitated vehicle routing problem", Systems Engineering Theory \& Practice, vol. 29, no. 2, pp. 159-166, 2009.

[14] A. Munawar, M. Wahib, M. Munetomo, and K. Akama, "Implementation and optimization of cGA+LS to solve capacitated VRP over Cell/B.E.", International Journal of Advanced Computer Technology, vol. 1, no. 2, pp. 16-28, 2009.

[15] T. Ning, C. Guo, L. Wang, "The shortest path optimization method using hybrid genetic algorithm", International Journal of Advanced Computer Technology, vol. 3, no. 6, pp. 305-311, 2011.

[16] T. Ning, and C. Guo, "Hybrid quantum algorithm for vehicle routing problem with time windows", Journal of Covnvergence Information Technology, vol. 7, no. 14, pp. 454-460, 2012.

[17] L. Wang, "Advances in quantum-inspired evolutionary algorithms", Control and Decision, vol. 23, no. 12, pp. 1321-1327, 2008.

[18] S. Zhang, T. Ning, and Z. Zhang, "A new hybrid ant colony algorithm for solving vehicle scheduling problem", International Journal of Advanced Computer Technology, vol. 4, no. 5, pp. 17-23, 2012 . 